\title{
Mast cells in the brain: Possibilities for physiological psychology
}

\author{
MICHAEL A. PERSINGER \\ Environmental Psychophysiology Lab, Laurentian University, Sudbury, Ontario, Canada
}

\begin{abstract}
Recent studies have demonstrated the presence of large numbers of mast cells (MCs) or similar cytological elements within the meninges and within perivascular tissue of brains from many mammalian species. The distributions of MCs within brain space appear to be a function of species, age, and possibly experience. Dense cytoplasmic granules within brain MCs appear to contain heparin, histamine, serotonin (in some species), protein complexes, and sulfomucopolysaccharides. MCs may account for almost all the histamine in the neonatal rat brain and up to $70 \%$ of this amine's content in the adult rat brain. The topographical, morphological, pharmacological, and immunological properties of MCs suggest their involvement in processes of interest to physiological psychologists. Detailed information concerning brain MC characteristics as well as possibilities for their functions are presented.
\end{abstract}

Mast cells (MCs), which may contain significant amounts of histamine, have been found within brain space. These cells have been considered to be longlived, mobile components of connective tissue (Bloom \& Fawcett, 1968; Copenhaver, Bunge, \& Bunge, 1971), but may compose a functionally independent intraorganismic system and only share the same space as the connective tissue matrix (Csaba, 1972). Abundant numbers of MCs are found typically around blood vessels and within the lung, uterus, spleen, liver, kidney, and heart. During disease conditions, anistropic concentrations of MCs may occur within many places, such as in lymphatic tissue.

Classical (nonneural) mast cells display densely granulated cytoplasmic patterns similar to that of the basophilic leukocyte in the blood. The granules contain sulfomucopolysaccharides like heparin (best known for its anticoagulation properties) to which the histamine is bound (Uvnas, 1974) as well as protein complexes, lipids, the ground substances hyaluronic acid or chondroitin sulfate (Watanabe, Watanabe, Ohishi, Aiba, \& Kageyama, 1974) and possibly serotonin in some species. When stained with thionin and toluidine blue in appropriate $\mathrm{pH}$ solutions, these granules demonstrate $\gamma$ metachromatic reactions (Kiernan, 1976) typified by pinkpurplish red colorations, whereas the cytoplasmic elements of other cells stain blue. In vitro and in vivo studies have demonstrated that these biopotent granules may be retained in the cell indefinitely or may be released explosively in response to a variety of chemical and mechanical stimuli. An excellent text on nonneural MCs has been prepared by Selye (1965).

\section{MAST CELLS IN THE CENTRAL NERVOUS SYSTEM}

\section{Characteristics and Methodology}

Traditionally, MCs have been regarded as virtually absent from the parenchyma of the CNS under normal conditions. Mast cells have been reported in the periphery of infarcts, around or within plaques associated with multiple sclerosis and in the vascular portions of syphilitic lesions of the human brain (Olsson, 1968), but these cases have been considered exceptional. However, accumulating evidence by Cammermeyer (1972, 1973), Campbell and Kiernan (1966), Dropp (1972, 1973, 1974, 1976), Flood and Krüger (1970), Ibrahim (1974a, b), Krüger (1974), Kelsall and Lewis (1964), and Persinger (1977) indicate that MCs or markedly similar cytological elements occur frequently and copiously within the normal brains of many mammalian species. According to Dropp (1976), MCs can be demonstrated in both the meninges and parenchyma of brains from 29 mammalian species, including man. MC distribution in brain space is highly variable and may be influenced by species differences and by procedures of fixation and staining.

In general, brain MCs share morphological features with MCs in other parts of the organism. In the adult mammalian brain, these cells are round, oval, or spindle-shaped with cytoplasmic granules that exhibit $\gamma$-metachromasia to appropriate stains. This experimenter has found good metachromatic and morphological demonstration of brain MCs in nonperfused rat brains quickly removed (with minimal mechanical trauma) following decapitation, fixed for 2-3 days in E.F.A. (90 parts $80 \%$ ethanol, 
5 parts $30 \%$ Formalin, and 5 parts concentrated glacial acetic acid), paraffin embedded, and stained with the thionin method after Lillie (Humason, 1972, p. 349); MCs in brain fixed with calcium Formalin and stained with toluidine blue 0 also show metachromasia. Alternative histological procedures have been discussed by Cammermeyer $(1972,1973)$ and Ibrahim (1974a). Fine-structure of brain MCs are similar to nonneural MCs (Flood \& Krüger, 1970) and display normal paracrystalline arrays with myelin-like figures.

Histochemical analyses have demonstrated the presence of histamine, serotonin, heparin, and related sulphomucopolysaccharides in brain MCs (Ibrahim, 1974a). MCs in the leptomeninges of the rat, mouse, and human brain histochemically display (by fluorescence) the presence of several enzyme groups, including phosphatases and peroxidases (Rosenblum, 1973a). According to Ibrahim (1974a), brain MCs, like their nonneural counterparts, display partial or total degranulation to MC stimulators. Ibrahim (1974a) and Ibrahim, Tenekjian, and Uthman (1976) have reported partial to full degranulation or anisogranulation in MCs within the brains of rabbits and cats following injections of compound 48/80 (a condensation product of paramethoxyphenethylmethylamine with formaldehyde frequently used in MC studies) or of hydrocortisone; rats displayed similar, but less intense, reactions. In context of stress studies, it is interesting that furclipped rabbits exposed to $5^{\circ} \mathrm{C}$ for $42 \mathrm{~h}$ displayed MC changes similar to those produced by hydrocortisone (Ibrahim, 1974a). Cammermeyer (1972) warns that some types of "degranulation" are artifacts of the microtoming procedure.

On the bases of histological and pharmacological reactions, Ibrahim $(1974 a, b)$ has considered the existence of two types of brain MCs. Type I brain MCs, whose morphological characteristics are considered identical with those of classical nonneural MCs by both light and electron microscopy, demonstrate metachromasia; these cells, according to Ibrahim (1974a), would be the ones reported by most investigators. Type II brain MCs display basophilic granules but do not react metachromaticly and contain larger amounts of lipids. Ibrahim (1974a) states that his Type II MCs traditionally have been called adventitial cells, pericytes, or perivascular cells, and may be lysosomal-like in nature. Type II cells display almost identical chemical constituents as Type I cells and undergo changes following hydrocortisone, C 48/80 and ionizing radiation treatments.

Technical disagreements have been voiced by Kiernan (1976), who argues that the cells observed by Dropp (1972) may be pericytes; these cells do not stain with Alcian blue or react metachromaticly with toluidine blue and therefore cannot contain heparin (Kiernan, 1976). However Dropp's criteria for MC counts have included metachromasia (Dropp, 1976; personal communication), and Ibrahim's (1974a) Type I cells also showed the typical tinctorial criterion. Again, the importance of fixation and staining techniques, and perhaps the premortem condition of the specimen, require more precise parametric evaluation. Heterogeneity in tinctorial staining capacity may also be an indicator of MC activity or functional involvement (Cammermeyer, 1973).

Mast cell numbers and localization are characterized by marked intraspecies and interspecies variation both within the leptomeninges and brain parenchyma. Within CNS parenchyma of adult organisms, MCs are invariably found individually or in clusters around blood vessels (Dropp, 1972, 1976; Kelsall \& Lewis, 1964) and have been demonstrated by electron microscopy to be located on the neuropil side of the blood-brain barrier (Dropp, 1974); these cells are not found around capillaries in the rat and cat according to Ibrahim (1974a), but do lie adjacent to blood vessels in the hedgehog according to Kiernan (1976). The latter experimenter found about $30 \%$ of hedgehog brain MCs scattered within neuronal and glial populations. Ibrahim (1974a) concludes that both Type I and Type II MCs are found predominantly within the CNS of the mouse, rat, hamster, and cat while primarily Type II MCs are found in the brains of guinea pigs, rabbits, dogs, monkeys, and man. However, Dropp $(1972,1976)$ reported numerous Type I MCs, which showed classical morphological and staining characteristics, in several of the latter species. Other morphological distinctions have been described by Cammermeyer (1972).

\section{Mast Cell Numbers and Brain Topography}

Numerical values for MCs within ostensibly normal brains of the more commonly used laboratory animals and of human subjects best demonstrate the large range of intraspecies and interspecies differences. The existence of intraspecies variation, especially within groups of animals bred under "similar" conditions, may be important for physiological psychologists since it could reflect the contribution of subtle experiential factors upon brain MC number and function. Alterations of these potent storehouses of CNS-effective chemicals would have important consequences upon behavior.

Dropp $(1972,1976)$ reported between 0 and 1,500 total MC numbers within the brains of squirrel monkeys, but found no brain MCs within five mulatta monkeys; three chimpanzees displayed 8,600, 15,470 and 69,570 total brain MCs. Thirteen cat brains analyzed by Dropp (1974) displayed total MCs ranging from 144 to 203,226 . The median value for seven meadow voles was 180 total brain MCs. 
The laboratory rat demonstrates a similar marked intraspecies variation. Total brain MCs ranged between 333 and 4,358 in 10 hooded rats and between 632 and 1,205 in 3 albino rats (Dropp, 1976). Unpublished data from my laboratory (T. H. Gryschuk, 1976; M. A. Persinger, 1976) indicates that some albino rat brains may show even greater MC concentrations. Twelve 100-day-old Wistar-strain male and female rats whose brains had been fixed in E.F.A. and stained with the Thionin method showed total MC (as identified by morphology and metachromasia) numbers ranging from 5,230 to 10,144 within the diencephalon alone. Total thalamic MCs from four 240-day-old normal male Wistar rats ranged from 3,328 to 4,050 .

Distributions of MCs within adult brain structures are not homogeneous. In albino rat brains, less than $1 \%$ of total MCs are found within major tract systems; the majority exist within gray matter around blood vessels. Less than $2 \%$ of total brain MCs occurred within cerebral cortices of the rodents studied (Dropp, 1972). The frontal and parietal cortices contained $63 \%$ and $34 \%$, respectively, of this small MC population (Dropp, 1976); the telencephalon, mesencephalon, metencephalon, and myelencephalon displayed $8.7 \%, 6.2 \%, 0.9 \%$, and less than $0.1 \%$ of the total MCs, respectively. Calculations from Dropp's (1972) data indicate that more than $80 \%$ of all brain MCs in rodents studied occurred within the thalamus, especially the dorsal-lateral portions. Unpublished data from my laboratory indicate the existence of a "standing wave" of MCs within the thalamus of 100- and 240-day-old Wistar rats with peak concentrations of 30 to $120 \mathrm{MCs} / 10-\mu \mathrm{m}$ section at the level of the habenula $( \pm 0.5 \mathrm{~mm})$; these concentrations decrease to zero as one moves rostrally or caudally from this point. Our analyses of structural localization are in agreement with Dropp (1976), who found major concentrations of MCs within the lateral nucleus, medial nucleus, posterior nucleus, ventral nucleus, and lateral geniculate. Whether these patterns reflect changes in MC function or in diencephalic geometry remains to be tested.

Carnivores also demonstrated MCs within diencephalic structures, but the specific nuclei of concentration differed from rodents (Dropp, 1976). Dropp (1976) noted that $17 \%$ to $68 \%$ of the total brain MCs in the chimpanzees studies occurred within the cerebral cortices, especially the frontal and parietal lobes; the percentages of cerebral cortical MCs only occurring in the frontal, parietal, and temporal lobes were $54 \%, 30 \%$, and $11 \%$, respectively. In 12 of the cats investigated by Dropp (1974), telencephalic MCs accounted for $12 \%$ to $90 \%$ $(\bar{X}=49 \%)$ of the total brain MCs. Ibrahim (1974a) found rich concentrations of MCs in cat cortices around the anterior rhinal sulcus. In many species, MCs are found concentrated in the olfactory bulbs, infundibulum, pituitary, pineal body, subfornical organ, facial nucleus, and area postrema (Dropp, 1976; Kelsall \& Lewis, 1964). In the latter structure, Cammermeyer (1972) has found up to 16,000 MCs near the ventricular surface in primates and carnivores, while rodents and lagomorphs demonstrated very few MCs; again no MCs were found in the mulatta monkeys studied. Asymmetric distributions of MCs have been noted in many mammalian brains, where up to $90 \%$ of total MCs concentrated in one particular side (Cammermeyer, 1973).

Normal human brains do not demonstrate MCs within major CNS structures. Unfortunately, there have been no systematic studies of brains from human subjects with functional disorders such as schizophrenia. Dropp's (1976, personal communication) latest investigations involved normal brains from 103 human subjects between 1 and 98 years of age. MCs were found only in the infundibulum, pineal body, area postrema, and supraoptic crest; four of the individuals demonstrated MCs within the subfornical organ while another three subjects showed MCs attached to choroid plexuses within the ventricles. Again large ranges in cell numbers were encountered. MCs within the adult pineal body ranged from only a few to 49,000 , while those in the infundibulum ranged from 0 to 7,000. Males demonstrated more MCs in the area postrema than females. Such sex differences have been found in some species (Krüger, 1974) but not in others (Cammermeyer, 1972; Kulshreshtha \& Dominic, 1972).

\section{Mast Cell Numbers and Aging}

MC numbers in the brain clearly change with the age of the animal. Most CNS structures show a decrease in MC numbers as a function of age, with the exception of the olfactory bulbs and some thalamic nuclei in the rat and in the hamster (Kelsall, 1966). In rodents, especially the albino rat, Dropp (1976) found an average of 7,356 total MCs within the brains of three 1-h-old rats, while the brains of three 1-week-old rats averaged 38,037. Three 1month-old rats averaged about 10,000 total MCs, while older animals (up to 2 years) averaged about 1,200 total brain MCs. During the first week of birth, brain MCs almost exclusively reside within the meningeal fabric. Krüger (1974) noted 6,000 MCs within the diencephalic leptomeninges alone. Thereafter, between Postnatal Days 7 and 10, MCs began to invaginate thalamic parenchyma. Eight 10-day-old $( \pm 2 \mathrm{~h}$ ) rats from eight different litters (eight pups/ litter) bred in my laboratory displayed between 113 and 717 total MCs within diencephalic tissue proper; the numbers of MCs within adjacent meninges ranged between 19,926 and 29,120 . 
MCs within human CNS structures demonstrate a similar pattern. According to Dropp (personal communication, 1976), MCs within human brain first appear: (1) in the pineal body around the 7 th month post partum, (2) in the infundibulum around the 14th month, and (3) in the area postrema around 3 years of age. In 25 human infundibulums from subjects between 1 and 39 years of age, an average of 2,500 MCs were counted (Dropp, 1976, in preparation). Infundibulums from individuals 40 to 79 years of age $(n=38)$ averaged about $700 \mathrm{MCs}$; similar age-related decreases were found within the pineal body. The number of individuals displaying brain MCs did not seem to vary with age.

Localization of MCs within particular brain structures is also age-dependent. While most MCs in adult rodent brains reside within diencephalic structures, Dropp (1976) found $63 \%$ to $76 \%$ of total brain MCs within the telencephalon of nine 1-week- to 1-monthold rats; thereafter the relative concentrations shifted to dicencephalic structures. A major area of concentration in 15-day-old rats involves the subfornical organ, which can display concentrations between 100 and $200 \mathrm{MCs} / 10-\mu \mathrm{m}$ section (Persinger, 1977). Such elevated concentrations are less obvious around the normal 20-day-old subfornical organ.

\section{CNS Mastocytogenesis}

The means by which MCs enter the CNS parenchyma are not clear. Ontologically one can follow the accumulating MC concentrations within the meningeal fabric and the later localization within diencephalic leptomeninges before closure of the cerebral hemispheres. Flood and Krüger (1970) assume that mature MC migration can occur only through the bloodstream and then through blood-vessel walls. There may be other alternatives since the 1:2.5 ratio of MC numbers found in 15- and 20-day-old thalamic tissue, respectively, was associated with a 2.7:1 ratio of MC numbers found in 15- and 20-day-old leptomeninges, respectively (Persinger, 1977). Although this parallel increase in thalamic MC numbers and decrease in meningeal $\mathrm{MC}$ numbers with age suggest a migrational property of MCs in the developing brain, other forms of proliferation must still be considered. Some authors suggest that brain MCs may differentiate from perivascular pericytes or endothelial cells (Ibrahim's Type II MCs), which are assumed to be multipotent in their morphological end points. Preliminary $\left(\mathrm{H}^{3}\right)$ thymidine uptake data reported by Ibrahim (1974a) indicate that mitotic division may occur in both Type I and II MCs during the first 2 weeks of life in the rat.

\section{CNS MCs During Pathlogical Conditions}

Excessive MC numbers within CNS tissue have been considered an occasional correlate of infectious or degenerative processes for decades. As mentioned, increase MC numbers or appearance of MCs in the brain have been noted in the vasculature of progressive or acute lesions and around plaques associated with multiple sclerosis (MS). Elevations and acid proteinase and acid phosphatase, two of the active constituents of human brain MCs, have been quantitatively measured in MS plaques (Hirsh, Duquette, \& Parks, 1976). Recently Olsson (1974) has confirmed the presence of MCs associated with the vascular walls in plaques distributed within the optic tracts of MS patients. MCs were not found in controls or in nondemyelinated areas of the chiasma in MS patients. Olsson suggests that MCs may participate in the as yet unexplained increased vascular permeability and focal edema around MS plaques, as well as the actual myelin degeneration. Olsson's ideas deserve attention in light of histamine's propensity to promote edema and heparin's influence on myelin degeneration (Enerback, Olsson, $\&$ Sourander, 1965).

Perivascular infiltration into CNS material by lymphocytes, plasma cells (which actively synthesize antibodies), and, on occasion, eosinophils have been correlated with lesions seen in experimental allergic encephalomyelitis, postvaccinal encephalitis, or even acute multiple sclerosis. According to Ibrahim (1974b), similar profiles can be elicited experimentally with ionizing radiation to which MCs vigorously react. Regions of focal hemorrhaging from radiogenic reactions and later bouts of demyelination seemed to concentrate within MC rich areas of gray matter. Ibrahim (1974b) has suggested that MCs are a primary initiator of the changes in vascular permeability proliferation of oligodendroglia and concomitant demyelination associated with all these conditions. That MCs participate actively in myelin degeneration, connective tissue response, and appearance of edema in peripheral nervous system neuropathies has been demonstrated (Enerback et al., 1965; Olsson, 1968).

\section{Responses to Experimental Manipulations}

Brain MCs can respond to various experimental treatments. As noted, Ibrahim (1974a, b) and Ibrahim et al. (1976) found partial or total MC degranulation or modified granule architecture a few days following injections of C $48 / 80(2 \mathrm{mg} / \mathrm{kg})$ or hydrocortisone $(200 \mathrm{mg} / \mathrm{kg})$; the responses were less apparent in the rat than in the rabbit, which reacted in a similar manner to cold exposure. Like body MCs, brain MCs respond to the blatant insults of ionizing radiation. Kelsall and Hunter (1970) have reported calcification of MC-rich thalamic regions of hamsters depleted of Vitamin $B_{6}$ for 15 weeks; this report is interesting in light of the tendency for discharged MC granules in the peripheral nervous system to attract, concentrate, and deposit iron and 
then calcium salts around themselves and on certain peripheral structures such as the vagus nerve (Selye, 1965). On the other hand, Cammermeyer (1972) found that neither the appearance nor the content of MCs in the area postremas of monkeys or cats were affected by treatment with cortisone (25 mg/kg over 25 days), L-histidine, reserpine, thyrotropic hormone, or castration; unfortunately, most of these experiments involved single subject designs. Experiments concerned with the effects of behavioral manipulations (such as conditioning schedules, anxiety paradigms, or other adrenal corticosteroid treatments upon MC numbers or changes) that would have implications for physiological psychology have not been reported in the literature.

MCs in the developing rat brain may be more responsive to experimental treatments. Using a splitlitter design, Persinger (1977) found that identification marking of the hind body surface of rat pups during Postnatal Days 5 to 20 was associated with significant and large reductions in thalamic MCs. Eight-, 15-, and 20-day-old pups that had been body marked demonstrated $50 \%$ to $70 \%$ reductions in MCs relative to eight nonmarked littermate controls. Daily injections of C $48 / 80(2 \mathrm{mg} / \mathrm{kg})$ were somewhat effective for reducing MC numbers, while daily 2-min electric shock applications were relatively ineffective with the sample size used. These results are interesting in context of the well-known effects of early experience, e.g., handling, marking, mothering effects, upon later emotional behavior (e.g., Denenberg \& Haltmeyer, 1967), histamine levels, and susceptibility to diseases (Stein, Schiavi, \& Camerino, 1976). In addition, MCs are known to concentrate in thalamic nuclei associated with propioceptive/tactual input and the Papez circuit. However, it is possible that the reduction in CNS MCs was a consequence of some toxic material in the marking fluid.

\section{Suggestions for Brain Mast Cell Functions}

The functions of MCs within the CNS are clearly unknown and research utilizing experimental designs is just beginning. Most speculation concerning MC functions are based upon their conspicuous localizations within brain space and upon their histochemistry. Ibrahim (1974a) speculates that brain MCs are involved with local haemodynamics, a view supported by the observation that MCs may influence vasomotor control of pial arterioles (Rosenblum, 1973b). A more specialized version of this hypothesis has been proposed by Campbell and Kiernan (1966), who suggested that MCs might modulate cerebral vasculature in the hedgehog during hibernation. Detoxification roles for MCs against exogenous and endogenous toxins have been envisioned by Ibrahim (1974a) as well as involvement with sulfate and lipid metabolism. The contributing role of MCs to myelination or demyelination (Olsson, 1974) has been implied from comparisons with PNS MC activity and MC proliferation following radiogenic lesions. Immunological roles of MCs have been suggested by Ibrahim (1974b), Kelsall (1966), and Schwartz (1975). The former two authors have concluded that MC-mediated dilation of brain microvasculature would allow passge of plasma proteins into tissue fluids. Similar "interface functions"' between brain and cerebrospinal fluid may involve MCs, since their copious presence in circumventricular structures, e.g., area postrema, pineal body, subfornical organ, etc., is well known (Cammermeyer, 1973).

\section{A BRIEF COMPARISON TO NONNEURAL MAST CELLS}

A brief description of nonneural MC functions may help us understand what possible roles these cells could have within the CNS. A vast literature already exists concerning the pharmacokinetics, histochemistry, species morphology, and significant stimuli for nonneural MCs (e.g., Csaba, 1972; Selye, 1965). In general, nonneural MCs seem to display structural and kinetic properties similar to cerebral MCs. However, body MCs have been demonstrated experimentally to be involved with important and sometimes severe biological consequences.

Perhaps the most well-known role of MCs outside the nervous system is concerned with the hypersensitivities, the most devastating form being total body or systemic anaphylaxis. Although the symptoms involved with anaphylaxis display species variability and depend upon the susceptible smooth muscle tissue, the reaction is characterized by muscle contraction, dilation of blood vessels, and edema (Barrett, 1970). The blood from animals undergoing anaphylactic shock demonstrates a delayed clotting time and is toxic to the skin of normal animals when administered intradermally. Typically, fatal shock is associated with edema and/or hemorrhaging in the lungs, heart, and interestingly, within the brain in many species, including man (Barrett, 1970). Nonlethal systemic anaphylaxis can be associated with symptoms of itching, headache, restlessness, respiratory/heart-rate increases, and gastrointestinal changes.

The precipitating conditions responsible for MCrelated hypersensitivities, including anaphylaxis, have been correlated with the release of histamine and heparin. Histamine is a well-known amine that can induce dilation of blood vessels, increased capillary permeability, and contraction of smooth muscle; heparin is a well-known anticoagulant and would facilitate histaminogenic effects upon humoral passage into perivascular tissue. It has been assumed 
that histamine produces changes by altering smooth muscle cells in the vascular system, which in turn leads to edema and erythema. Contraction of bronchiolar musculature, one of the many consequences of this activity, would promote asphyxia. Compound $48 / 80$, the potent MC degranulator, will induce the synthetic duplicate of anaphylactic shock in animals. Appropriate applications of antihistamines can eliminate these reactions. However, antihistamines will not totally eliminate anaphylactic reactions in species (e.g., the rat) whose MCs also contain serotonin.

The predisposing factor associated with anaphylactic shock involves an immunological reaction; this reaction requires a certain latency such that immunoglobulin synthesis progresses to some criterion level. Essentially, it is assumed that plasma cells are responsible for the synthesis of immunoglobulins that are released and circulate through the blood while homocytotropic immunoglobulins attach to mast cells. Following injection of antigen, the mast-cell-bound antibodies engage in a reaction that releases MC granules into extracellular fluid (Uvnas, 1974) and influences smooth muscle/blood changes. The expulsion or degranulation procedure can be complete within a few seconds, although the actual release of histamine from the granule in the extracellular fluid is dependent upon cations such as $\mathrm{Na}^{+}$(Lagunoff, 1972) and most probably $\mathrm{Ca}^{+}+$ (Foreman \& Mongar, 1972). Calcium ions are known to be required for anaphylactic release of histamine from guinea-pig lung, human leukocytes, and rat peritoneal MCs.

Mast cell lysis can be achieved by a number of other immunological and chemical techniques. Both IgG and IgE fractions can initiate MC extrusions under optimal conditions. In addition to the mechanisms involving combinations of antigen and antibody on cell surfaces, certain kinds of neutrophils that have engulfed antigen-antibody complexes release compounds that are mastocytolytic (Barrett, 1970). MCs, aptly described as migratory unicellular "glands" (Bloom \& Fawcett, 1968), may be involved with delayed hypersensitivities that involve perivascular infiltration of lymphocytes, but the data are not clear. Liberation of histamine promotes blood flow and local inflammatory edema that dilutes irritant stimuli and may activate phagocytosis of other cell types.

Histamine within nonneural mast cells displays a slow turnover but is readily released by compound 48/80; exogeneous histamine can prevent this effect (Ambrus \& Ambrus, 1972; Antila \& Pohto, 1972). Non-mast-cell histamine turns over rapidly and is not released by this compound. It is most important that degranulated mast cells (e.g., by compound $48 / 80$ ) still retain their ability to store granules and release histamine upon a second challenge (Yamasaki \& Sugiyama, 1972). Mast cells also respond to a number of drugs relevant to psychophysiological studies. Clinical dosages of anti-rheumatic agents such as hydrocortisone and phenylbutazone inhibit histamine release by anaphylactic action. Mast cells are apparently damaged by antihistamine drugs like chlorpromethazine and chlorpromazine (Vugman \& Gomez Rodrigues, 1974). More detailed information on body mast cells has been compiled by Seyle (1965).

\section{PSYCHOPHYSIOLOGICAL IMPLICATIONS OF BRAIN MCs}

In summary, mast cells (1) are found in large numbers within the brains of many mammalian species, (2) are calculated to contain large percentages of histamine, (3) can demonstrate quick degranulation responses, (4) maintain the ability to degranulate more than once, and (5) are reactive to psychotropic drugs. These properties suggest the following possible functions of MCs within the brain.

\section{Mast Cells As Major Reservoirs of Brain Histamine}

MCs within the brain and nonneural structures have been demonstrated to contain sulphomucopolysaccharides and histamine. The former substances involve chondroitin sulfate and heparin sulfate which have been strongly implicated as binding complexes for catecholamines within vesicles of neural tissue (Pycock; Blaschke, Bergqvist, \& Uvnas, 1975). Histamine has been considered a CNS transmitter candidate and has been demonstrated within a variety of CNS structures (Green, 1970) and may display a "circuitry" similar to that of CNS catecholamines (Schwartz, 1975). The degree to which MCs contribute to brain histamine would have significant implication for brain models.

Relationships between MC numbers and histamine concentration in various nonneural tissues have been shown many times (Green, 1970). Quantitative parallels between histamine content in the pineal body and MC numbers have been reported, although histamine occurs in species not demonstrating MCs in this structure. In rat brain, the age-related peak and decline in MC numbers are reflected in whole brain histamine concentrations which achieve maxima in the 2- to 6-day-old rat brain but rapidly decrease after Postnatal Day 10 (Krüger, 1974).

Simple calculations indicate that MCs could contain significant proportions of whole brain histamine in the rat. Assumptions are usually made that a brain MC contains histamine concentrations similar to that of a nonneural MC, e.g., peritoneal MC, which can contain between $7 \mathrm{pg} / \mathrm{cell}$ and $32 \mathrm{pg} /$ cell (Selye, 
1965) of this .substance; to this experimenter's knowledge, no direct measurements of histamine concentrations within CNS MCs have been reported. If each brain MC did contain $10 \mathrm{pg}$ of histamine, then a 100-day-old rat containing 2,000 MCs would sequester $20 \mathrm{ng}$ of brain histamine; a rat containing 7,000 MCs could involve $70 \mathrm{ng}$ of brain histamine. Whole-brain total concentrations of histamine range from $50 \mathrm{ng}$ to $250 \mathrm{ng}$, depending upon assay technique (Green, 1970). Assuming total adult rat brain histamine values of $70 \mathrm{ng}$ made by Green and Erikson (1964), as an example, then brain MCs could account for a significant, if not a major, portion of total brain histamine. Similarly, in the 1-week-old rat brain, which contains about $300 \mathrm{ng}$ of histamine and averages 38,037 MCs (Dropp, 1976), MCs could contain almost all brain histamine. Similar calculations have been made by Dropp $(1972,1976)$ and Krüger (1974).

It is interesting that drugs which influence nonneural MCs also alter histamine levels in the adult cat brain. According to a review by Green (1970), reserpine $(0.5$ to $10 \mathrm{mg} / \mathrm{kg})$ did not affect MCs in the pituitary (which has been assumed to contain MCs that can be discharged with $C 48 / 80$ ) but reduced histamine in the hypothalamus and thalamus by $40 \%$ and $60 \%$, respectively. CPZ elevated histamine in the hypothalamus and medial thalamus by $50 \%$ and $32 \%$, respectively, while iproniazid treatment for 5 days $(25 \mathrm{mg} / \mathrm{kg})$ increased histamine in the hypothalamus by $46 \%-74 \%$ and in the medial thalamus by $28 \%$. The significant modification in hypothalamic histamine could be explained by the presence of MCs in this species. It should be remembered that the hypothalamus contains only moderate amounts of histamine relative to total brain content.

A mastocytogenic source of rat brain histamine of this magnitude is contrary to present assumptions concerning this amine's localization. Green (1970) assumed no MCs within the CNS (with the exception of the hedgehog) and concluded that almost all histamine resided in neural tissue primarily within particulate matter, but this was before more recent documentation of MC occurrence within CNS tissue. Biochemical analyses by Schwartz, Lampert, and Rose (1972) and others seem to confirm Green's conclusion, since brain histamine is primarily found in synaptosomes or in microsomal fractions, although its distribution within subcellular fractions from whole brain differs from that of norepinephrine, serotonin, and slow-reacting substances. With the exception of a bimodal distribution of histamine in the guinea pig brain, where one fraction has been identified with MCs and/or granules from disrupted MCs (Michelson \& Dowe, 1963), the contribution of MCs to brain histamine has been considered minimal or nonexistant.

However, a unitary neurogenic or synaptogenic concept of rat brain histamine is also not commensurate with the available data. For example, most cerebral amines display low concentrations at birth, when synapses are few. Histamine, in contrast to the others, displays levels that are six times higher than in the adult rat brain (Schwartz, 1975); yet the activity of histidine decarboxylase, the synthesizing enzyme of histamine, is very low in the neonate brain but elevated in the adult brain. The half-life of ${ }^{3} \mathrm{H}$ histamine injected at birth in the neonate brain is four days, quite unlike the half-lives of less than $1 \mathrm{~h}$ known to occur in the adult brain, and more like the value for MC-rich tissues (Schwartz, 1975). Moreover, Martres, Baudry, and Schwartz (1975) have reported a release of histamine from neonatal brain slices stimulated by two mast-cell degranulators-C $48 / 80$ and polymixine $B$.

The solution to the discrepancy may lie within a combination of source multiplicity and methodological interpretation. It is possible, as with the guinea pig, that different parts of the brain store histamine in different ways; gross analyses of the brain would obscure this feature. This view is consistent with the partial effects of histamine synthesis inhibitors which induce an immediate decrease in histamine within a fraction of the brain but do not influence a compartment representing about $50 \%$ of the total, even with dosage increases. Another example of this condition can be seen in the pituitary, where C 48/80 did not affect the concentration of histamine in the anterior lobe but reduced it markedly in the neurohypophysis, a result which suggests that histamine is bound primarily to MCs in the latter structure (Green, 1970).

Secondly, the existence of histamine within synaptosomes or within microsomal fractions does not necessarily exclude MC fragments to which histamine-heparin-sulphomucopolysaccharide-protein complexes would be attached. Often the proof that the majority of histamine is within neural tissue has been based upon: (1) electron microscopic verification of materials from nonrich MC portions of the rat brain such as the cerebral cortices (e.g., Kataoka \& De Robertis, 1967); (2) the mere presence of histamine in fractions containing particles resembling nerve endings; or (3) the relative uptake of histamine by nerve endings in isolated microsomal preparations. MC granules range from 0.2 to $0.5 \mu \mathrm{m}$ within the range of materials typically collected by microsomal preparations and display structural-chemical similarities to neural fragments (Bacchawat, Balasubramanian, Balasubramanian, Singh, George, \& Chandrasekaran, 1972; Ibrahim, 1974a). An empirical verification of substances from $\mathrm{MC}$ granules in whole rat-brain microsomal fractions would demand a reevaluation of the role of MCs in brain histamine.

In a recent minireview, Schwartz (1975) presents 
clear data of such verification and constructs a tentative synopsis of differential properties of histamine stores in neurons and mast cells in the rat brain. Schwartz concludes that a bimodal distribution of histamine clearly exists for rat brain even when controls for methodology have been taken into account. While histamines in synaptosomes was found in the $\mathrm{P}_{2} \mathrm{~B}$ portion of the crude mitochondrial fraction, another major concentration of histamine was found in the crude nuclear $\left(P_{1}\right)$ fraction or the $P_{3}$ microsomal fraction. The latter fraction of histamine, which sedimented with the crude nuclear pellet, demonstrated a low ratio of histidine decarboxylase activity to endogeneous histamine as well as a slow turnover of ${ }^{3} \mathrm{H}$-histamine. These characteristics are typical of the neonatal rat brain and typical mast cells. In fact, when typical mast cells were isolated from the rat peritoneal cavity and added to the brain homogenate before differential centrifugation, the histamine was localized almost entirely in the crude nuclear pellet, suggesting again that histamine in this fraction of rat brain is held by mast cells. Certainly, a mastocytogenic source of brain histamine would demand a reevaluation of the role of these cells and histamine in the rat brain.

\section{MCs as Gateways to Brain-Blood Immuno- logical Reactions}

The conspicuous position of brain MCs around blood vessels implies a potential function in aberrant interactions between blood and brain fluids. Since brain MCs contain histamine, heparin, and other compounds, sudden release of these substances could increase the permeability of the blood-brain barrier through direct or indirect modifications of the endothelial cells within the blood vessels assumed to contribute to this interface and would influence behaviors associated with portions of the brain predominantly influenced. In the rat, for example, where MCs congregate primarily around blood vessels within thalamic nuclei associated with sensory input and emotional behavior, one would expect modifications in these functions. Such contacts between normally separated fluids could contribute to serious complications. Two possibilities will be discussed here: (1) acute schizophrenic-like episodes, and (2) demyelination.

For some time now, some forms of schizophrenia have been postulated to be autoimmune diseases (Burch, 1964; Heath, 1971). The known elevations of certain plasma globulins, e.g., $\alpha$-2-globulins, serum hemolysis, and membrane permeability alterations in schizophrenic patients (Gottlieb, Frohman, $\&$ Beckett, 1971), are compatible with the concept that autoantibodies may develop in response to intracellular substances leaking into the blood stream, the kinetics of which could be influenced by substances released by perivascular brain MCs.
The contribution of histamine-related processes to behavioral pathology has been suspected since the time that: (1) allergic reactions were infrequently found in psychotics, and (2) it was noted that some patients alternated between allergies and psychosis (Eiduson, Geller, Yuwiler, \& Eiduson, 1964). Indeed, elevations of blood histamine levels are known to occur in schizophrenics, especially acute cases, and more recently, Heath (1971) reported marked similarities between electroencephalographic changes induced by known "psychosis-inducing" immunoglobulins and histamine when these compounds were injected directly into the brains of primates. These observations superficially support a MC involvement, since chlorpromazine, a major tranquilizer, displays antihistamine properties and has deleterious effects upon nonneural MC function.

However, MCs do not seem to occur normally within major CNS parenchyma, such as the cerebral cortices, of human subjects. If the hypothesis of MC involvement is correct, then the existence of some mastocytogenic factor within the schizophrenic brain should be found during acute episodes. As noted, MCs are capable of migration. MC numbers are known to fluctuate in the brains of monkeys (Ibrahim, 1974b) during conditions simulating delayed hypersensitivities and have been observed to invade major CNS tissue during autoimmune-related diseases (Olsson, 1974).

Another obvious aspect of brain MCs in the rat is the peak concentration numbers around the first week after birth. It is during this time that the brain develops programs to recognize its own selfconstituents, the "self-antigens" vs. the "non-selfantigens" according to Berry and Riches (1974). These processes later prevent antibody reactions against the organism's own proteins. Many foreign materials, if injected during this period, will not elicit an immune response. After this period, the body is no longer tolerant to antigenic constituents and even those associated with CNS myelin, which begins developing around Postpartum Day 10 when the time for induction of tolerance has passed, will elicit an immune response. Conceivably, the presence of MCs in the brain during this critical period allows for the differential reaction to substances that are recognized as "self-antigens" or "non-self-antigens." Nonneural MCs have been postulated as immunological memory banks by Padawer (1974).

The most direct evidence for MC involvement with brain-related immunological reactions (Schwartz, 1975) involves the processes of demyelination (Ibrahim, 1974b; Olsson, 1974) or failures of remyelination, since the latter may be immunogenic as well (Berry \& Riches, 1974). MCs are known to be intimately involved with myelin-destructive processes in the PNS and have been reported to appear around vascular portions of demyelinating brain areas in 
MS and syphylitic lesions. Heparin is known to stimulate phospholipase in plasma and the liberation of fatty acids from lipoproteins has been linked to MCs in demyelinating regions (Olsson, 1968).

Ibrahim (1974b) used radiogenic sources to induce demyelination in monkey brains and noted increased MC numbers associated with intense perivascular mononuclear cell infiltrates (primarily lymphocytes and plasma cells) within areas later prone to demyelination and within the leptomeninges. Similar patterns have been noted in brains affected by experimental allergic encephalomyelitis and acute MS. Although the loci of MS lesions vary markedly within brain white matter, there is an unexplained tendency for their appearance in periventricular areas and around veins, especially during the early phases of the disease (Forster, 1973). Both these observations could support the involvement of MCs in the demyelinating process.

In fact, Ibrahim (1974b) has argued that MCs may actually initiate the demyelinating sequence, since: (1) they contain hydrolytic enzymes that could damage myelin directly or indirectly by altering vascular permeability, and (2) delayed lesions, associated with perivascular and leptomeningeal infiltrates of mononuclear cells and myelin damage similar to irradiation damage, have appeared in the brains of rats, cats, and rabbits following MC damage by compound $48 / 80$ and high dosages of hydrocortisone. Predictably, antihistamines can attenuate the early increase in vascular permeability in the irradiated rat and prevent nonneural MC disruption in the irradiated hamster (Smith, 1958).

The effect observed by Ibrahim (1974b) that is most germane to recurrent diseases involved the delayed radiation damage to myelin that was associated with proliferation of hypertrophied MC numbers months after the initial irradiation, a response which Ibrahim suggests could be due to a delayed hypersensitivity reaction to some early MC constituent. This observation indicates that once MCs have been proliferated and sensitized in a particular brain region, the exposure of the prone organism to some subsequent MC-degranulating stimulus, perhaps an adrenal corticosteroid-related conditioning paradigm, e.g., "anxiety," could precipitate another bout of degranulation and concomitant demyelination. Since Ibrahim argues that a single intense dose $(200 \mathrm{mg} / \mathrm{kg})$ of hydrocortisone can degranulate MCs but cortisone therapy $(\sim 1 \mathrm{mg} / \mathrm{kg}$ / day) can ameliorate acute demyelinating conditions, the interactions between MCs and these steroids are not elementary. One would suspect that the therapeutic procedure would slowly degranulate susceptible MCs or prevent their sudden release to antigen. Corticosteroids have been reported to prevent the anaphylactic release of nonneural MCs, and, according to Barrett (1970), antecedent de- granulation of nonneural MCs will prevent their response to consequent antigen exposures. At this time, it is not clear why single dosages of chemical structures that attenuate demyelinating bouts at low concentrations can precipitate antagonistic reactions at higher concentrations. Brain MCs are known to increase in numbers following low doses of ionizing radiation but to decrease in numbers following more massive dosages.

The precise mechanisms by which MCs participate in the above disease processes must still be isolated; no doubt many of these will involve immunogenictype stimuli whose primary source will be derived from unusual brain-blood interactions. However, there are other possible triggers that may involve operations more familiar to the physiological psychologist. For example, can excessive neuroexcitation in MC-rich areas contribute to the alterations in MC function? Metabolic consequences of excessive and prolonged neuroelectrical and glial activity are associated with significant changes in blood-brain barrier interactions as greater energetic demands are required. In the "normal" rat, where most MCs concentrate within nuclei associated with subcortical integration of sensory input and of emotional behavior, one would suspect that excessive stimulation of these areas could alter proximal MC function. In the "normal" chimpanzee, where the majority of MCs are found within the frontal and parietal cortices, one would suspect that metabolic consequences of excessive electrical activity in these lobes associated with the appropriate behavior, e.g., inhibition and somesthetic input, would stimulate MC changes. "Abnormal" brains associated with unusual MC concentrations in various brain regions would be influenced by behavioral situations associated with these areas. Degranulation of nonneural MCs have been observed following antidromic stimulation of cutaneous nerves (Kiernan, 1971).

\section{Are Brain MCs Subject to Conditioning and Reinforcement Histories?}

Since MCs can degranulate more than once, the possibility arises that "conditioned MC degranulation" could occur. The occurrences of respondentlike conditioning in physiological systems have been reported many times. Injections of isotonic saline (or other stimuli) that have been paired with the presence of insulin can produce conditioned hypoglycemia (Balagura \& Harrell, 1975) and the pairing of electric shock with the shock chamber can induce conditioned eosinopenia in the blood (Gollender, 1967). It is interesting that conditioned taste aversions induced by pairing saccharin with ionizing radiation (Kimeldorf \& Hunt, 1965; Levy, Carroll, Smith, \& Hofer, 1974), to which MCs strongly react, can be eliminated by antihistamines (Levy, et al., 1974). 
Ideally, conditioned brain MC degranulation would involve some humoral UCS, e.g., such as an antigen-antibody stimulus (that elicits a UCR, e.g., degranulation) paired with a neuroelectrical event or its consequences such that the presentation of the latter stimuli (CSs) would induce a CR (modified degranulation). Another possibility would involve MC degranulation as a consequence of conditioning. In this model, the presentation of an aversive UCS elicits a UCR. If another stimulus occurs before the aversive UCS, then an exaggerated "CR" can be elicited. For example, if electric shock is preceded by a tone, the presentation of the tone by itself can elicit elevations ("CR") in adrenal corticosteroids that are even higher than the initial response (UCR) to the UCS. Such paradigms, which characterize conditioned suppression or conditioned anxiety situations, would be associated with marked elevations in chemical structures known to influence MC activity. Exposure of prone organisms to these paradigms could induce changes that might precipitate MC leakage.

Conditioned activity of MCs, although not necessarily an anaphylactic-like all-or-none pattern, becomes attractive when one considers the various psychogenic stimuli allegedly responsible for reports of headaches, allergic reactions, asthma attacks, skin irritations, abdominal pains, arthritic complaints, and related symptoms. Usually these reactions are reserved for various placebo labels, yet they can be treated by salicylates and antiallergic compounds that work (at least in part) by their antihistamine reactions (Wilson \& Schild, 1968). MCs are known to occur around extracranial and intracranial (dural) blood vessels, a frequent source of "headaches," and within other parts of the body associated with the above symptoms. Whether degranulation of brain MCs could induce extrusion of granules in their nonneural counterparts has not been established.

A final feature of brain MCs involves their numerical variability within members of the same species, an observation that implies the role of experience. Can specific conditioning or reinforcement histories influence the numbers of MCs within specific brain regions and hence contribute to the MC reactions noted previously? Could certain early conditions influence MC numbers/activity and predispose the organism to later CNS-related disease conditions? Such possibilities would seem likely in light of the Ibrahim's (1974a) reports of MC changes associated with cold exposure and the influence of marking on preweaning MC numbers in rats (Persinger, 1977). The latter effect would appear important, since such stimulus operations profoundly influence later emotional behavior and histaminerelated processes in rats. In addition, significant amounts of young brain histamine may be bound to
MCs (Schwartz, 1975). These possibilities, although capable of generating many more interesting possibilities, must await the results of experimental testing and empirical evaluation.

\section{REFERENCES}

Ambrus, M. S., \& Ambrus, J. L. Comparative study on the effect of mast cell disintegrating agents on clearing factor lipase activity in various species and sexes. Research Communications in Chemical Pathology and Pharmacology, 1972, 4, 449-456.

Antila, R., \& Pohto, P. Inhibition of histamine release from mast cells by fluoride ions in vivo. Annales Medicinae Experimentalis et Biologie Fenniae, 1972, 50, 78-81.

BachHawat, B. K., Balasubramanian, K. A., Balasubramanian, A. S., Singh, M., George. E., \& Chandrasekaran, E. V. Chemistry and metabolism of glycosaminoglycans of the nervous system. Advances in Experimental Medicine and Biology, 1972, 25, 51-71.

Balagura, S., \& Harrell, L. E. Neuroendocrine conditioning: Conditioned feeding after alterations in glucose utilization. American Journal of Physiology, 1975, 228, 392-396.

BARRETT, J. T. Textbook of immunology. Saint Louis: Mosby, 1970. Pp. 185-191.

BERRY, M., \& Riches, A. C. An immunological approach to regeneration in the central nervous system. British Medical Bulletin, 1974, 30, 135-140.

BLOoM, W., \& FAWCETT, D. W. A textbook of histology. Toronto: Saunders, 1968. Pp. 152-153, 161-162.

BuRCh, P. R. J. Schizophrenia: Some new aetiological considerations. British Journal of Psychiatry, 1964, 110, 818-828.

CAMMERMEYER, J. Mast cells and postnatal topographic anomalies in mammalian subfornical body and supraoptic crest. Zeitschrift für Anatomie und Entwicklungsgeschichte, 1973, 140, 245-269.

CAMMERMEYER, J. Mast cell in the mammalian area postrema. Zeitschrift für Anatomie Entwicklungsgeschichte, 1972, 139, 71-92.

Campbell, D. J., \& Kiernan, J. A. Mast cells in the central nervous system. Nature, 1966, 210, 756-757.

Copenhaver, W. M., Bunge, R. P., \& Bunge, M. B. Bailey's textbook of histology. Baltimore: Williams and Wilkins, 1971. P. 115.

CsabA, G. Regulation of mast-cell formation. Budapest: Akademiai Kiado, 1972.

Denenberg, V. H., \& HAltme Yer, G. C. Test of the monotonicity hypothesis concerning infantile stimulation and emotional reactivity. Journal of Comparative and Physiological Psychology, 1967, 63, 394-396.

DROPP, J. J. Mast cells in the central nervous system of several rodents. Anatomical Record, 1972, 174, 227-239.

Dropp, J. J. Mast cells in the mammalian brain. American Zoologist, 1973, 13, 514.

Dropp, J. J. Mast cells in the cat brain. Proceedings of the Pennsylvania Academy of Sciences, 1974, 48, 45-49.

DropP, J. J. Mast cells in the mammalian brain. I. Distribution. Acta Anatomica, 1976, 94, 1-21.

Eiduson, S., Geller, E., Yuwiler, A., \& Eiduson, B. T. Biochemistry and behavior. Toronto: Van Nostrand, 1964.

Enerback, L., Olsson, Y., \& Sourander, P. Mast cells in normal and sectional peripheral nerve. Zeitschrift fur Zellforschichte, 1965, 66, 596-608.

Flood, P. R., \& KRüGER, P. G. Fine structure of mast cells in the central nervous system of the hedgehog. Acta Anatomica, 1970, 75, 443-452.

Foreman, J. C., \& Mongar, J. L. The role of the alkaline earth ions in anaphylactic histamine secretion. Journal of Physiology, 1972, 224, 753-769.

Forster, F. M. Clinical neurology. Saint Louis: Mosby, 1973. Pp. 91-95. 
Gollender, M. Eosinophil and avoidance correlates of stress in anterior cingulate cortex lesioned rats. Journal of Comparative and Physiological Psychology, 1967, 64, 40-48.

Gottlieb, J. S., Frohman, C. E., \& Beckett, P. G. S. The current status of the $\alpha-2$ globulin in schizophrenia. In H. E. Himwich (Ed.), Biochemistry, schizophrenia and affective illness. Baltimore: Williams \& Wilkins, 1971. Pp. 153-170.

Green, J. P. Histamine. In A. Lajtha (Ed.). Handbook of neurochemistry. (Vol. IV) Control mechanisms in the nervous system. New York: Plenum Press, 1970. Pp. 221-250.

Green, H., \& ERICKson, R. W. Effect of some drugs upon rat brain histamine content. International Journal of Pharmacology, $1964,3,315-320$.

HeAth, R. G. An antibrain globulin in schizophrenia. In H. E. Himwich (Ed.), Biochemistry, schizophrenia and affective illness. Baltimore: Williams \& Wilkins, 1971. Pp. 171-197.

Hirsch, H. E., Duquetre, P., \& Parks, M. E. The quantitative histochemistry of multiple sclerosis plaques: Acid proteinase and other acid hydrolases. Journal of Neurochemistry, 1976, 26, 505-512.

Humason, G. Animal tissue techniques. San Francisco: Freeman, 1972. P. 349.

IBRAHIM, M. Z. M. The mast cells of the mammalian central nervous system. Part 1: Morphology, distribution and histochemistry. Journal of the neurological sciences, 1974, 21, 431-478. (a)

IBRAHIM, M. Z. M. The mast cells of the mammalian central nervous system. Part 2: The effect of proton irradiation in the monkey. Journal of the Neurological Sciences, 1974, 21, 479-499. (b)

Ibrahim, M. Z. M., Tenekjian, V., \& Uthman, M. A. E. The effect of compound $48 / 80$ on the perivascular granular cells (neurolipomastocytes) of the rabbit brain. Anatomical Record, 1976, 184, 434.

KATAOKA, K., \& DeRobertis, E. Histamine in isolated small nerve endings and synaptic vesicles of rat brain cortex. Journal of Pharmacology and Experimental Therapy, 1967, 156, 114-125.

Kelsall, M. A. Aging on mast cells and plasmacytes in the brain of hamsters. Anatomical Record, 1966, 154, 727-740.

Kelsall, M. A., \& Hunter, R. E. Mast cells and calcification in the brain of pyridoxine deficient hamsters. Anatomical Record, 1970, 166, 330 .

Kelsall, M. A., \& Lewis, P. Mast cells in the brain. Federation Proceedings, 1964, 23, 1107-1108.

KIERnAN, J. A. Degranulation of mast cells following antidromic stimulation of cutaneous nerves. Journal of Anatomy, 1971, 111, 349-350.

KIERNAN, J. A. A comparative survey of the mast cells of the mammalian brain. Journal of Anatomy, 1976, 121, 303-311.

KIMELDORF, D. J., \& HunT, E. L. Ionizing radiation: Neural function and behaviour. New York: Academic Press, 1965. Pp. 261-263.

KRÜGER, P. G. Demonstration of mast cells in the albino rat brain. Experientia, 1974, 30, 810-811.

Kulshreshrha, A., \& Dominic, C. J. Mast cells in the hypothalamus and the pituitary gland of the musk shrew, Suncus murimus, L. (Insectivora). Journal of Animal Morphology and Physiology, 1972, 19, 23-27.

LAGUNOFF, D. The mechanism of histamine release from mast cells. Biochemical Pharmacology, 1972, 21, 1889-1896.
Levy, C. J., Carroll, M. E., Smith, J. C., \& Hofer, K. G. Antihistamines block radiation-induced taste aversions. Science, 1974, 186, 1044-1045.

Martres, M. P., Baudry, M., \& Schwartz, J. C. Histamine synthesis in the developing rat brain; evidence for multiple compartmentation. Brain Research, 1975, 83, 261-275.

Michaelson, J. A., \& Dowe, G. The sub-cellular distribution of histamine in brain tissue. Biochemical Pharmacology, 1963, 12, 949-956.

Orsson, Y. Mast cells in the nervous system. International Review of Cytology, 1968, 24, 27-70.

Orsson, Y. Mast cells in plaques of multiple sclerosis. Acta Neurologica Scandinavica, 1974, 50, 611-618.

Padawer, J. The ins and outs of mast cell function. American Journal of Anatomy, 1974, 141, 299-302.

Persinger, M. A. Preweaning body marking reduces brain mast cell numbers in rats. Behavioral Biology, 1977, in press.

Pycock, C., Blaschke, E., Bergqvist, U., \& Uvnas, B. On the possible involvement of sulphomucopolysaccharides in the storage of catecholamines within the central nervous system. Acta Physiologica Scandinavica, 1975, 95, 373-382.

Rosenblum, W. I. A possible role for mast cells in the production of pial vasopasm. Journal of Neuropathology and Experimental Neurology, 1973, 32, 173. (a)

Rosenblum, W. I. A possible role for mast cells in controlling the diameter of arterioles on the surface of the brain. Brain Research, 1973, 49, 75-82. (b)

SchwarTz, J. C. Histamine as a transmitter in the brain. Life Sciences, 1975, 17, 503-518.

SChwartz, J. D., LAMpart, C., \& Rose, C. Histamine formation in the rat brain in vivo: Effects of histidine loads. Journal of Neurochemistry, 1972, 19, 801-810.

SELYE, H. The mast cells. Washington: Butterworths, 1965.

SмITH, D. E. Influence of antihistaminics on mast cell disruption following X-irradiation. Proceedings of the Society for Experimental Biology and Medicine, 1958, 97, .872-874.

Stein, M., Schiavi, R. C., \& Camerino, M. Influence of brain and behavior on the immune system. Science, 1976, 191. 435-440.

Uvnas, B. Histamine storage and release. Federation Proceedings, 1974, 33, 2172-2176.

Vugman, I., \& Gomez Rodrigues, J. M. The inhibitory effect of toluidine blue and methylene blue on rat mast cell damage by promethazine, chlorpromethazine and chlorpromazine. Its reversal by glucose. Experientia, 1974, 30, 1178-1180.

Watanabe, S., Watanabe, K., Ohishi, T., Aiba, M., \&

KAGEYAMA, K. Mast cells in rat alveolar septa undergoing fibrosis after ionizing radiation. Laboratory Investigation, 1974, 31, 555-567.

Wilson, A., \& Schild, H. O. Applied pharmacology. Boston: Little, Brown, 1968. Pp. 357-358.

Yamasaki, H., \& SugiYama, K. Histamine release from isolated rat mast cell in metal ion-free medium. Japanese Journal of Pharmacology, 1972, 22, 325-327.

(Received for publication September 7, 1976; revision accepted November 29,1976 .) 